\title{
COMMISSION 20: POSITIONS AND MOTIONS OF MINOR PLANETS, COMETS AND SATELLITES
}

\author{
(POSITIONS ET MOUVEMENTS DES PETITES PLANETES, DES COMETE: \\ ET DES SATELLITES)
}

\section{PRESIDENT: H. Rickman}

VICE-PRESIDENT: E.L.G. Bowell

ORGANIZING COMMITTEE: K. Aksnes, J.-E. Arlot, A. Carusi, A. Lemaîitre, B.G. Marsden V.A. Shor, D.K. Yeomans \& J.-X. Zhang

SECRETARY: G.B. Valsecchi

\section{INTRODUCTION}

This Comm. 20 triennial report is prepared as a short summary of the scientific progress in the fields of interest, where, by necessity, only a few highlights are to be mentioned. On the Comm. 20 webpage, the interested reader may find a more complete list of recent references to the relevant literature. Its URL at the time of writing (October 1999) is http://www . astro. uu.se/IAU/c20/, but mirror sites are expected to have been set up at the time of appearance of these Transactions.

The Comm. 20 Working Group on Satellites has prepared a separate triennial report, immediately following this one, where the format is rather like in previous IAU Transactions, the work being described in considerable detail.

Annual reports of the Minor Planet Center can be found in the IAU Information Bulletins. From these, and from Sect. 3 of this report, it is obvious that the recent developments in observational as well as computational activities have implied an enormous increase of the work load for this Center. The problems created by this are presently under discussion, and solutions are sought, whereby the funding of the MPC can be increased and/or its tasks shared in order to secure a future operation that avoids the loss of its current, high scientific standard.

\section{MEETINGS}

During the period covered by this report there has been a number of meetings and schools relevant to the dynamics of small solar system bodies, namely:

- IAU Colloquium 165 Dynamics and Astrometry of Natural and Artificial Celestial Bodies, 1-5 July 1996, Poznań (Poland);

- Asteroids, Comets, Meteors 96, 8-12 July 1996, Versailles (France);

- NATO Advanced Study Institute The Dynamics of Small Bodies in the Solar System: A Major Key to Solar System Studies, 29 June-12 July 1997, Maratea (Italy);

- Dynamics of Comets and Asteroids and their Roles in the Earth History, 14-18 August 1997, Hikone (Japan);

- IAU Colloquium 172 Impact of Modern Dynamics in Astronomy, 6-11 July 1998, Namur (Belgium);

- IAU Colloquium 173 Evolution and Source Regions of Asteroids and Comets, 24-28 August 1998, Tatranská Lomnica (Slovakia). 


\section{MINOR PLANETS}

Observational activity on minor planets experienced a tremendous surge during the triennium. As had been anticipated, several new CCD search programs for NEOs (near-earth objects) came on line, joining the older Spacewatch and NEAT (Near Earth Asteroid Tracking) programs. One of the new programs, LINEAR (Lincoln Laboratory Near Earth Asteroid Research), alone made almost three times as many observations of minor planets as were produced by all of the world's observatories during the previous triennium! Furthermore, the number of observations made worldwide during this triennium was nearly one-and-a-half times the number included when the magnetic tape with the complete set of observations was last issued in 1995.

The increase in observational activity is obviously also reflected in the rate of accumulation of orbits. February 1999 saw the numbering of the ten-thousandth minor planet; by the end of June 1999, the number of numbered minor planets was already 10986 , a 56-percent increase since June 1996. Together with some 13000 other objects recorded at two or more oppositions and single-opposition orbits of varying degrees of quality, the total number of entries in the official files in mid-1999 was 52346.

Provisional designations were given to 61666 initially unidentified minor planets, nearly three times the number of designations during the preceding triennium. In 1998 the subscripted number in the designations exceeded 100 for the first time--and it did so in four different halfmonths, as well as in two halfmonths in the first half of 1999.

Some 320 different observatories were involved in making astrometric observations during the triennium, although only some 120 would typically be active during a given month. The observatories were located in some 35 different countries, and more than 70 percent of them were owned and operated by amateur astronomers. Fourteen observing programs, listed in Table 1, contributed more than 7500 observations during the triennium, and they were collectively responsible for 88 percent of the observations.

Table 1. Leading observing programs

\begin{tabular}{||l|rll||}
\hline Program & Number & Telescope & P.I. \\
\hline LINEAR & 725277 & $1.0-$ m GEODSS & G. H. Stokes \\
Spacewatch & 126143 & $0.91-$ m reflector & R. S. McMillan \\
LONEOS & 103493 & $0.59-$ m Schmidt & E. L. G. Bowell \\
NEAT & 43333 & $1.0-$ GEODSS & E. F. Helin \\
Xinglong & 35240 & $0.60-$ m Schmidt & J. Zhu \\
ESO & 33744 & $1.0-$ - Schmidt & E. W. Elst, C.-I. Lagerkvist \\
ODAS & 27742 & $0.90-$ m Schmidt & A. Maury, G. Hahn \\
Klet & 19764 & $0.57-$ m reflector & J. Tichá \\
Catalina & 17792 & $0.41-$ m Schmidt & S. M. Larson \\
Oizumi & 13929 & $0.25-$ m reflector & T. Kobayashi \\
Višnjan & 13295 & $0.41-$ m reflector & K. Korlević \\
Ondřejov & 10300 & $0.65-$ m reflector & P. Pravec \\
Woomera & 8843 & $0.30-$ m reflector & F. B. Zoltowski \\
Prescott & 7659 & $0.46-$ m reflector & P. Comba \\
\hline
\end{tabular}

Generally from a single-night detection of a suspected NEO candidate, a brief ephemeris is routinely placed in "The NEO Confirmation Page" (NEOCP) in the World Wide Web at http://cfa-www.harvard.edu/iau/NEO/ToConfirm.html. Observers at a couple of dozen sites are now regularly monitoring this site, and some of them often report followup observations within a matter of hours of the discovery, thereby allowing revisions of the ephemeris and further follow-up observations. As soon as the orbit computation has reasonably stabilized, a Minor Planet Electronic Circular is issued (provided that the object 
is still an NEO or otherwise unusual) and the object is removed from the NEOCP. For the first time, two objects found to have retrograde orbits failed to show cometary activity, even when subjected to close scrutiny. These were $1999 \mathrm{LD}_{31}$ and $1999 \mathrm{LE}_{31}$, LINEAR discoveries made just four nights apart, and they had orbital inclinations of $160^{\circ}$ and $152^{\circ}$, respectively.

Since the vast majority of the NEOs have no possibility of colliding with the Earth during the foreseeable future, it has been convenient to define a subset of "potentially hazardous asteroids" (PHAs). These are minor planets that are currently able (under simplified assumptions) to pass within $0.05 \mathrm{AU}$ of that of the Earth and have absolute magnitudes brighter than 22 (i.e., to include objects as small as 200 meters across). The 91 PHAs known in June 1996 precisely doubled to 182 in June 1999.

The topic of assessing the possibility and the probability of an impact by a known object, in terms of the observational information available at particular times, became one of some interest during this triennium, and various techniques for dealing with this problem have been developed. As this field is very actively debated, there have been only very few publications (Sitarski, Acta Astron. 49, 103-112, 1999; Milani, Chesley and Valsecchi, Astron. Astrophys. 346, L65-L68 1999). From the point of view of Commission 20, it is important that particular attention be paid to objects for which there exists the possibility of specific impact events during the next 50-100 years, even when the impact probability is some orders of magnitude smaller than the "background" level, i.e., the probability of the Earth's being hit in a given year by an undiscovered object of comparable size.

On the theoretical side, Milani (Icarus 137, 269-292, 1999) has begun a series of papers on the asteroid identification problem. He has also continued, with Knežević, his work on the problem of proper elements for main belt asteroids (Celest. Mech. and Dynam. Astron. $71,55-78,1998)$.

A number of studies have addressed the problem of the regularity of motions in the main belt; the surprising new result is that "slow" chaos, i.e. chaos whose manifestation takes a time comparable to the age of the solar system, is very frequent (Milani, Nobili and Knežević, Icarus 125, 13-31, 1997; Froeschlé, Gonczi and Lega, Planet. Space Sci. $45,881-886,1997)$ and is due either to high-order mean motion resonances (Holman and Murray, Astron. J. 112, 1278-1293, 1996) or to the presence of three-body mean motion resonances (Murray, Holman and Potter, Astron. J. 116, 2583-2589, 1998; Nesvorný and Morbidelli, Astron. J. 116, 3029-3037, 1998). In particular, the asteroids of the inner belt undergo chaotic diffusion, that ultimately can make their orbits Mars-crossing (Morbidelli and Nesvorný, Icarus 139, 295-308, 1999).

Numerical explorations of the long-term motion of asteroids in the region of the inner planets have started to uncover the dynamical structure of that region (Michel and Froeschlé, Icarus 128, 230-240, 1997; Michel, Planet. Space Sci. 46, 905-910, 1998; Migliorini et al., Science 281, 2022-2024, 1998).

At the end of the previous triennium the count of TNOs stood at 37, with 18 (including Pluto) observed at multiple oppositions and 19 at only a single opposition. The multiple-opposition objects were mainly plutinos (objects in 2:3 mean-motion resonance with Neptune) and cubewanos (low-eccentricity orbits with mean distances 43-46 AU), together with single objects at the $3: 4$ and 3:5 resonances. At the end of this triennium the ratio of the single-opposition to multiple-opposition objects was 121 to 54 . While it is exciting to see that improved search techniques are turning up TNOs in much greater numbers than before, the slow rate at which objects are recovered at a second opposition is rather disturbing. As one might expect, there has been some augmentation of the types of orbits represented, although the plutinos and cubewanos still dominate.

It is now clear that there are TNOs with orbits that are much larger than those of the cubewanos, although there is as yet no indication that these larger orbits have their perihelion distances more than a few astronomical units beyond Neptune, even though one of these objects, $1999 \mathrm{DG}_{8}$, seems currently to be located around $60 \mathrm{AU}$ from the sun. Best known among these scattered-disk objects is $1996 \mathrm{TL}_{66}$, still the only one recorded at more 
than one opposition. There also seem now to be two known objects, $1996 \mathrm{TR}_{66}$ and 1997 $\mathrm{SZ}_{10}$, in 1:2 resonance with Neptune, with their perihelia close to Neptune's orbit.

Another interesting point is the distribution of the TNOs in inclination. Five multipleopposition objects have inclinations in excess of $25^{\circ}$, the highest inclination being $31^{\circ} .6$ for $1996 \mathrm{RQ}_{20}$, which otherwise seems to be a mid-range cubewano.

The scattered-disk objects are effectively an extension of the centaurs, which have unstable orbits in the realm of the giant planets, in some cases with their aphelia beyond Neptune. During the triennium eight new centaurs were added, bringing the total to 14, of which half have been observed at more than one opposition.

For what concerns the dynamics, studies of slow chaotic phenomena in the transneptunian belt have been carried out by Morbidelli (Icarus 127, 1-12, 1998), and Ip and Fernández (Astron. Astrophys. 324, 778-784, 1997) investigated the injection of objects from the 2:3 mean motion resonance into Centaur orbits, that can eventually lead to shortperiod comets.

\section{COMETS}

Theoretical and numerical studies have dealt with various aspects of the dynamics of comets. The level of performance reached by scientific workstations, coupled with the use of faster algorithms for the integration of the motion of bodies in chaotic orbits, have allowed the computation of orbital evolutions over timescales comparable with the age of the solar system. In particular, the long path connecting the trans-neptunian regions with the Jupiter family has been explored by Levison and Duncan (Icarus 127, 13-32, 1997), without the need to increase the masses of the perturbing planets that had been criticized, among others, by Valsecchi and Manara (Astron. Astrophys. 323, 986-998, 1997).

Emel'yanenko and Bailey have addressed the problem of the capture of Halley-type comets from the near-parabolic flux (Mon. Not. R. Astron. Soc. 298, 212-222, 1998), finding that small- $q$ Oort cloud comets contibute most to the Halley-type flux, and Wiegert and Tremaine (Icarus 137, 84-121, 1999) have studied the evolution of long-period comets, and tested various fading laws, in order to elucidate the problem of the paucity of old comets in the observed sample. Chambers (Icarus 125, 32-38, 1997) found an interesting dynamical difference between Halley-type and long-period comets, consisting in the absence, among the latter, of the mean motion resonances that are frequently found among the former.

Two other outstanding problems in cometary dynamics have been dealt with. The first is the low efficiency of implantation of comets into the Oort cloud in the first phases of the evolution of the solar system; Fernández (Icarus 129, 106-119, 1997) finds that the efficiency problem can be overcome if the solar system formed in a much more perturbed environment, like a molecular cloud or an open cluster.

The second long-standing problem is that of the origin of comet 2P/Encke, whose aphelion is currently well decoupled from the orbit of Jupiter. Steel and Asher (Mon. Not. R. astron. Soc. 281, 937-944, 1996) suggest that the action of nongravitational forces several times stronger than those observed for this comet can be sufficient to obtain the current orbit starting from one in the Jupiter family.

An interesting recent development is the work of Gronchi and Milani (Celest. Mech. and Dynam. Astron. 71, 109-136, 1998; Astron. Astrophys. 341, 928-935, 1999) aimed, in the long run, at constructing a theory of (temporary) proper elements for objects on planet-crossing orbits.

Hans Rickman 